\title{
Aberrant signaling in T-cell acute lymphoblastic leukemia: biological and therapeutic implications
}

\author{
B.A. Cardoso, A. Gírio, C. Henriques, L.R. Martins, C. Santos, A. Silva and J.T. Barata \\ Unidade de Biologia do Cancro, Instituto de Medicina Molecular, Faculdade de Medicina, Universidade \\ de Lisboa, Lisboa, Portugal
}

Correspondence to: J.T. Barata, Unidade de Biologia do Cancro, Instituto de Medicina Molecular, Faculdade de Medicina, Universidade de Lisboa, 1649-028 Lisboa, Portugal

Fax: +351-2-1799-9524. E-mail: joao_barata@fm.ul.pt

T-cell acute lymphoblastic leukemia (T-ALL) is a biologically heterogeneous disease with respect to phenotype, gene expression profile and activation of particular intracellular signaling pathways. Despite very significant improvements, current therapeutic regimens still fail to cure a portion of the patients and frequently implicate the use of aggressive protocols with long-term side effects. In this review, we focused on how deregulation of critical signaling pathways, in particular Notch, PI3K/Akt, MAPK, Jak/ STAT and TGF-ß, may contribute to T-ALL. Identifying the alterations that affect intracellular pathways that regulate cell cycle and apoptosis is essential to understanding the biology of this malignancy, to define more effective markers for the correct stratification of patients into appropriate therapeutic regimens and to identify novel targets for the development of specific, less detrimental therapies for T-ALL.

Key words: T-cell acute lymphoblastic leukemia; Notch; PI3K/Akt; MAPK; Jak/STAT; TGF-ß

Research supported by Fundação para a Ciência e a Tecnologia (FCT; POCI/SAU-OBS/58913; PTDC/SAU-OBD/69974) and Associação Portuguesa Contra a Leucemia, awarded to J.T. Barata. A. Gírio has a post-doctoral fellowship, A. Silva, B.A. Cardoso and C. Henriques have PhD fellowships, and L.R. Martins has a BI fellowship, all from FCT. C. Santos had an IEFP fellowship. With the exception of the senior author, J.T. Barata, the names of the authors appear in alphabetical order.

Received December 11, 2007. Accepted March 31, 2008

\section{Introduction}

Acute lymphoblastic leukemia (ALL) is the most common childhood malignancy, resulting from the clonal expansion of lymphoid progenitors that have undergone malignant transformation at distinct stages of differentiation. Approximately $15 \%$ of the cases are of T-cell origin ( $T$ ALL) (1). Cell-autonomous lesions are certainly at the origin of T-ALL. However, microenvironmental factors are also believed to contribute to T-ALL expansion $(2,3)$. Both cell-intrinsic defects and external stimuli frequently converge on the activation of key 'pro-oncogenic' intracellular pathways. In this review, we focus on the Notch, PI3K/Akt, MAPK, Jak/STAT and TGF- $ß$ signaling pathways, crucial for the survival and proliferation of the leukemic blasts
(Figure 1). We present a brief overview of these pathways, summarize their involvement in T-ALL and discuss their potential as therapeutic targets.

\section{Notch}

Activation of Notch pathway relies on the interaction of the single pass transmembrane receptor Notch with a Delta/Serrate ligand of a neighboring cell. This interaction activates a cascade of proteolytic cleavages that ultimately leads to activation of Notch-target genes and changes in the transcriptional program. In mammals, four Notch receptors are described (Notch1-4), which are homologues of Drosophila notch, and five Notch ligands (Delta-like-1,3 and 4 and Jagged1-2) that are homologues 
of Drosophila delta/serrate genes, respectively (4).

The Notch receptors are heterodimers that non-covalently interact at the cell membrane surface. The extracellular subunit $\left(\mathrm{N}^{\mathrm{EC}}\right)$ interacts with a ligand and the transmembrane subunit $\left(\mathrm{N}^{\mathrm{TM}}\right)$, which also contains the cytoplasmic effector domains of the receptor. The Notch ligands are all expressed at the cell surface as monomers or homodimers (5).

Activation of Notch receptors occurs upon ligand binding through the EGF-like repeats, present both in ligands and in the receptors. This interaction induces a conformational change in the Notch receptor that leads to two successive proteolytic cleavages, by metalloprotease tumor necrosis factor converting enzyme and by the $\gamma$ secretase complex. Consequently, intracellular Notch domain (ICN) is released and translocates into the nucleus. Nuclear ICN is capable of binding to CSL (CBF1, Suppressor of Hairless, and Lag1) and form a ternary complex with Mastermind-like proteins (MAML1-3), leading to the transcriptional activation of Notch-target genes. The best characterized Notch-target genes include Notch-signal modulator Deltex1, Hairy/enhancer of split (HES1-5) and Hairyrelated (HRT, HEY) gene family members. These genes are up-regulated upon Notch signaling and have been implicated in the modulation of proliferation, differentiation and survival (4).

Notch involvement in T-ALL was first described in three patients with $\mathrm{t}(7 ; 9)(q 43 ; q 34.3)$, which juxtaposes the TCRß locus to the C-terminal coding region of the Notch1 gene. This translocation leads to the expression of a truncated, cytoplasmic form of Notch1 receptor with constitutive activity (6). Human truncated Notch1 was shown to be highly oncogenic, since transduction of mouse bone marrow cells with retroviruses encoding the corresponding sequence of Notch1 leads to the development of T-cell leukemia with high penetrance in the transplanted animals (7), and the effect is synergistic with those of other known oncogenes, including c-Myc and E2A-PBX1 $(8,9)$. Despite these striking observations, $t(7 ; 9)(q 43 ; q 34.3)$ is very rare, accounting for less than $1 \%$ of T-ALL cases (10). However, the involvement of Notch1 in the pathogenesis of this disease was strongly supported by the discovery that more than $50 \%$ of T-ALL cases harbored mutations in the Notch 1 alleles, resulting in the constitutive activation of the pathway (10). The mutations were found in two different sites in the $\mathrm{N}^{\mathrm{TM}}$ subunit, the HD domain and the PEST sequence. The HD mutations enhance $\gamma$-secretase-dependent proteolytic cleavages in the receptor, whereas the PEST

Figure 1. Schematic representation of the signaling pathways that appear to contribute to proliferation and survival of T-cell acute lymphoblastic leukemia (T-ALL) cells. Several signaling components have been implicated in the regulation of T-ALL. The chimeric TEL-Jak2 protein is expressed in T-ALL due to $t(9 ; 12)(p 24 ; p 13)$ and is capable of phosphorylating STAT1 and STAT5, which could promote T-ALL proliferation. The Notch pathway was shown to be constitutively activated in more than $50 \%$ of T-ALL patients. The loss of Smad3 expression was demonstrated in some T-ALL cases, and may synergize with other oncogenic events in the development of this malignancy. Constitutive activation of the PI3K/Akt pathway was demonstrated in T-ALL cell lines and our own data indicate that it is highly frequent in primary leukemia cells. This essentially results from PTEN inactivation by different mechanisms. Mutations in MAPK family members have not been reported in T-

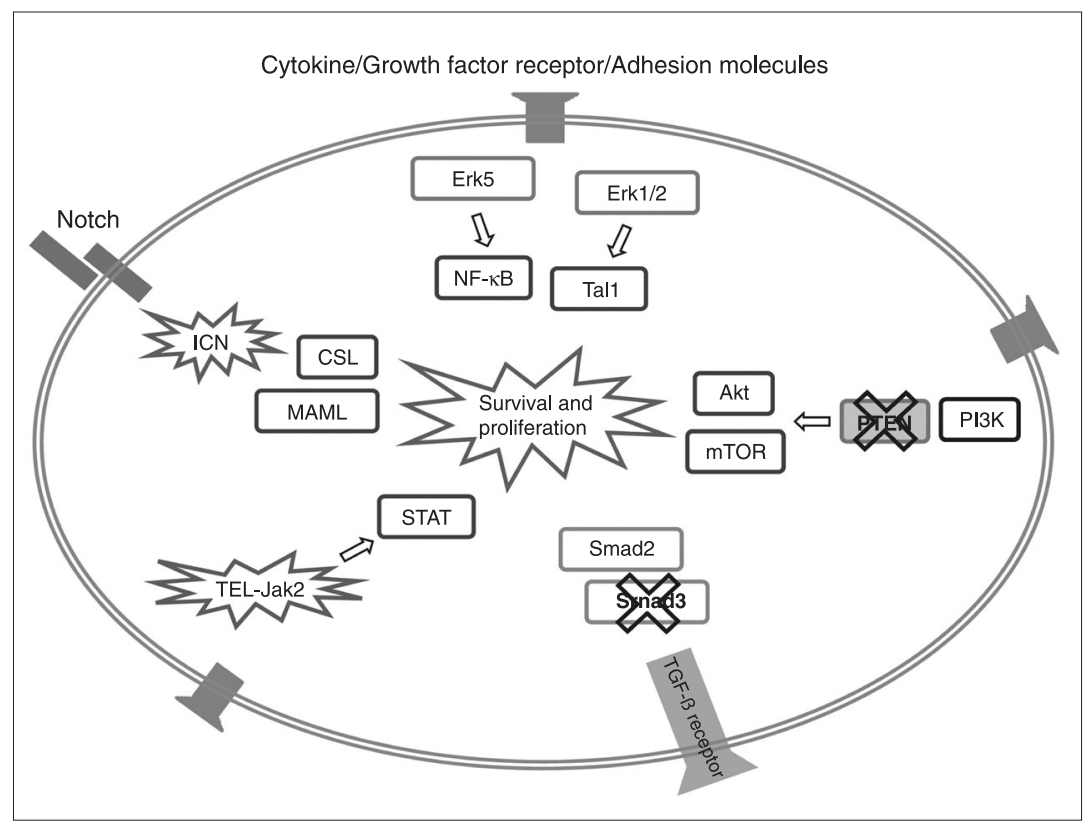
ALL. However, tumor-supportive signals from the microenvironment likely activate Erk1/2 and Erk5. Erk $1 / 2$ phosphorylates the T-cell oncogene Tal1, and Erk5 phosphorylates NF- $\mathrm{kB}$, contributing to the viability and proliferation of leukemic cells. Similarly, extracellular cues (cytokines/chemokines, growth factors, adhesion molecules, Notch ligands, etc.) leading to the activation of Notch or Jak/STAT pathways are probably present within the leukemic milieu. Although microenvironmental signals are unlikely to trigger the oncogenic process, they appear to contribute to the maintenance/proliferation of the malignant clones. ICN = intracellular Notch domain: CSL = CBF1, Suppressor of Hairless, and Lag1; MAML = Mastermind-like proteins. 
mutations result in premature stop codons and consequent deletion of the PEST domain that increases the halflife of ICN (11). Similar mutations were found in T-ALL mouse models (12). In addition, non-mutational Notch1 activation seems to be an early event in the development of T-ALL in mouse models (13), and recent work identified c-Myc as a Notch1-target gene highly up-regulated in TALL and critical for Notch1-mediated leukemogenesis (14).

Notch3 receptor has also been implicated in T-cell leukemogenesis. A transgenic mouse model that expresses Notch $3^{\text {ICN }}$ in thymocytes develops aggressive T-cell leukemias of immature phenotype (15). This phenotype was shown to be dependent on constitutive preTCR signaling that induces activation of NF- $\kappa B$, inhibition of E2A activity and Tal1 expression, contributing to the survival and proliferation of these tumors (16). However, it is not known how Notch3 can promote aberrant signaling capable of leukemogenesis, since it is normally expressed throughout Tcell ontogeny.

In contrast to Notch1 and -3 , Notch2 and -4 have not been associated with T-ALL, and the only Notch ligand implicated in the disease so far was Dll4. Retroviral-mediated transduction of bone marrow cells with DIl4 induced a lymphoproliferative disease of $\mathrm{CD} 4^{+} \mathrm{CD} 8^{+}$double-positive $\mathrm{T}$ cell precursors. Transplantation of these cells resulted in the establishment of aggressive leukemia in recipient mice (17).

As mentioned previously, Notch signaling is dependent on a cascade of proteolytic cleavages, one of which is catalyzed by $\gamma$-secretase complex in the $\$ 3$ cleavage site. In fact, inhibition of Notch signaling by $\gamma$-secretase inhibitors (GSI), already available for treatment of Alzheimer disease, was shown to prevent growth and induce apoptosis of T-ALL cell lines in vitro $(10,18)$. Consequently, GSI were regarded as very promising therapeutic tools for TALL (19). Nevertheless, the efficiency of these drugs in patients demands confirmation, since T-ALL cells seem to be frequently resistant to GSI treatment in vitro, perhaps due to activation of PI3K/Akt pathway (20).

\section{PI3K/Akt}

Phosphatidylinositol-3OH-kinase (PI3K) pathway regulates various cell functions including proliferation and apoptosis, through activation of different downstream effectors, of which the most prominent is the Ser/Thr kinase Akt (also called PKB, Protein Kinase B). At the cell membrane $\mathrm{PI} 3 \mathrm{~K}$ has the ability to phosphorylate phosphatidylinositol 4,5-biphosphate (PIP2) at the 3-position of the inositol ring, generating phosphatidylinositol 3,4,5-triphosphate (PIP3). PIP3 act as second messengers and serve as membrane anchors for proteins with a pleckstrin homology $(\mathrm{PH})$ do- main, such as Akt and PDK1. The colocalization of Akt and PDK1 at the cell membrane favors Akt phosphorylation and consequent activation (21). Akt generates anti-apoptotic and pro-proliferative signals through the inhibitory phosphorylation of Bad, GSK3, Forkhead (FOXO1) and caspase-9, through the activating phosphorylation of mTOR, and through the release of transcriptional factors such as NF- $\kappa B(21,22)$. The tumor suppressors PTEN and SHIP dephosphorylate PIP3, acting as negative regulators of the PI3K/Akt pathway (21).

In T-ALL, the PI3K/Akt pathway is activated by growth factors present in the leukemia milieu that signal through cytokine receptors $(2,23)$. Moreover, T-ALL cell lines present constitutively phosphorylated forms of Akt independently of external growth factors (20). In addition, NF- $\mathrm{KB}$, a downstream target of PI3K/Akt, is constitutively activated in some primary T-ALL samples (24). Nevertheless, no activating mutations of PI3K and/or Akt have been described in T-ALL. It has been suggested that PI3K/Akt pathway over-activation results from PTEN protein downregulation and/or SHIP mutations $(25,26)$. However, results from our lab show that non-deletional inactivation of PTEN is a major contributor to hyperactivation of PI3K/Akt in primary T-ALL samples (Silva A, Yunes JA, Cardoso BA, Martins LR, Jotta PY, Abecasis M, Nowill AE, Leslie NR, Cardoso AA, Barata JT, unpublished data). PTEN mutations resulting in protein truncation have been identified more frequently in T-ALL cell lines established from relapsed patients $(30.4 \%)$ than in diagnostic clinical specimens (5.2\%), which suggests that PTEN deletion is a late event in human T-ALL (25). In contrast, recent studies using mouse models have shown that PTEN deregulation is important at early stages of leukemogenesis. In these models, PTEN expression was shown to be essential to maintain the hematopoietic stem cell pool and to prevent leukemia development $(27,28)$.

As mentioned previously, PI3K activates several downstream signal transduction pathways including mTOR. Rapamycin, a specific inhibitor of mTOR, stimulates apoptosis of pediatric T-ALL cells $(29,30)$, reverses the chemoresistance of Notch1-overexpressing leukemic cells (31) and restores the normal function of hematopoietic stem cells from PTEN-deleted mice (27).

Several therapeutic strategies targeting PI3K pathway are now in development. In a number of studies the in vitro use of specific PI3K pharmacologic inhibitors, such as LY294002 and wortmannin, and natural compounds with PI3K inhibitory capacities, such as resveratrol, increased apoptosis and arrested the cell cycle in T-ALL cells $(32,33)$. $\mathrm{PI} 3 \mathrm{~K}$ inhibition is also important to abolish chemoresistance to drugs used in current therapeutic regimens or that 
are being tested in clinical trials (20).

\section{MAPKs}

Mitogen-activated protein kinases (MAPKs) are a family of evolutionary conserved Pro-directed Ser-Thr kinases, which play a central role in transducing extracellular cues into a variety of intracellular responses including the modulation of cell proliferation, differentiation and apoptosis (34). The activity of MAPKs depends on the phosphorylation state of the Thr and Tyr residues in a TXY motif within their kinase domain. This canonical motif is also used to classify MAPKs into three sub-families. $X$ corresponds to Glu in the extracellular signal-regulated kinases (Erks), to Pro in the c-Jun N-terminal kinases (JNK 1, 2, and 3) and to Gly in the p38 ( $\alpha, \beta, \gamma$, and $\delta$ isoforms) (34). The Erk subfamily comprises not only the widely studied Erk1 and Erk2, but also the more recently identified Erk5, containing an extended C-terminal domain that confers to it additional properties (35). External stimuli, consisting mainly, but not exclusively, of growth factors in the case of Erks and stress stimuli in the case of JNK and p38 (36), activate membrane receptors, which then turn on a cascade of activating phosphorylations. These proceed from an MAPKKK (MAPK kinase kinase) to an MAPKK (MAPK kinase) and finally to the MAPK. Once activated, MAPKs phosphorylate a number of cytosolic and nuclear substrates, leading to the diverse biological effects mentioned above (37).

Despite extensive evidence implicating activation of Erk pathway in cancer progression, the involvement of MAPKs in T-ALL is not clearly established. Neither JNK nor p38 have been shown to be altered in T-ALL. In contrast, there is evidence that Erks may contribute to T-ALL biology. T-cell Acute Leukemia 1 (Tal1) is a basic helix-loop-helix transcription factor involved in early hematopoiesis and normally down-regulated upon T-cell lineage commitment. Alterations in the tal1 gene, leading to its overexpression, are the most common genetic lesion in T-ALL (38). Tal1 oncogenic effects are thought to be mediated, at least in part, by heterodimerization and consequent inactivation of E2A/HEB tumor suppressor proteins (39). Aberrant activation of the Tal1 protein by phosphorylation via Erk1 has also been described in the human T-ALL cell line Jurkat (40). This modification has been suggested to positively regulate Tal1 transactivation potency (41) and may therefore link Erks to T-ALL leukemogenesis. However, there are no reported Erk-activating mutations in T-ALL as far as we know. Notwithstanding, activation of Erk1/2 has been observed in TALL cells on stimulation with IL-7. In contrast, normal T-cells do not appear to activate Erk1/2 in response to IL-7 (22). Despite this clear difference, the exact biological role of activation of Erk pathway remains to be elucidated, since it does not apparently have any major consequences on proliferation or viability of T-ALL cells (2).

In Jurkat T-ALL cells, Erk5 was also recently demonstrated to drive the phosphorylation, directly or via Rsk1, of the $\mathrm{p65/RelA}$ subunit of NF- $\mathrm{kB}$. As a consequence, $\mathrm{p65/}$ RelA is maintained in the nucleus, where it acts as a transcription factor for survival genes. This decreases apoptosis and has been proposed to be essential for the survival of leukemic T-cells (42).

Since both Erk1/2 and Erk5 have been reported to contribute to the survival of leukemic T-cells, they are logical candidates for therapy. Their inactivation with the use of pharmacologic inhibitors of upstream molecules, such as PD98059 and U0123 for MEK (MAPKK) and BAY-43-9006/ Sorafenib for Raf (MKKK), as well as their down-regulation through antisense approaches, are currently being studied for cancer treatment, although not specifically in the context of T-ALL (43). Therapies involving the activation of the stress-induced MAPKs have also been described. The glucocorticoid and common therapeutic agent dexamethasone has been shown to induce apoptosis of the human T-ALL cell line CCRF-CEM by activating p38, which in turn induces transcription of bim, a pro-apoptotic Bcl-2 family member (44). Further, arachidonic acid has been shown to have an anti-proliferative effect in Jurkat cells mediated at least in part by the activation of JNK (45).

\section{Jak/STAT}

The evolutionarily conserved Janus kinase-signal transducer and activator of transcription (Jak/STAT) pathway plays an important role in biological processes such as apoptosis, differentiation, proliferation and cellular immune responses, mediated by growth-factors and cytokines (46). Binding of cytokines results in cell surface receptor oligomerization and activation of the Jak family of tyrosine kinases (47). Activated Jaks phosphorylate the cytoplasmic domain of the receptor, thereby creating docking sites for STATs, which are phosphorylated by Jaks and consequently dimerize and migrate to the nucleus where they regulate gene transcription (47). Although rarely mutated, STATs are frequently overexpressed and hyperactivated by alterations in upstream signaling pathways, participating in oncogenesis through up-regulation of genes encoding apoptosis inhibitors ( $\mathrm{Mcl}-1, \mathrm{Bcl}-\mathrm{x})$ and cell cycle regulators (cyclins D1/D2, c-Myc) (48).

In pediatric $\mathrm{T}-\mathrm{ALL}$, the genetic juxtaposition between JAK2 and the ETS-family member TEL results from $\mathrm{t}(9 ; 12)(\mathrm{p} 24 ; \mathrm{p} 13)$ chromosomal translocation, and originates a constitutively active TEL-Jak2 chimeric protein (49). 
Strikingly, TEL-Jak2 transgenic mice develop fatal leukemia, displaying a selective expansion of CD8-positive Tcells in blood, lymph nodes, thymus, spleen, and bone marrow, and also invasion of non-hematopoietic organs by leukemic T-cells (49). These observations support the notion that TEL-JAK2 is an oncogene in vivo. Interestingly, the expression of a tyrosine-phosphorylated TEL-Jak2 protein correlates with activation of STAT1 and STAT5 in leukemic tissues (49). Moreover, TEL-Jak2, in addition to activating STAT5, appears to associate with Shc and Grb2 and induce activation of Erk2 (50).

Activation of STAT5 in T-ALL may arise from other mechanisms. For example, the cryptic $t(9 ; 14)(q 34 ; q 32)$, associated with deletion of p16 and expression of HOX11, was recently reported in a T-ALL patient, and results in the aberrant expression of a constitutively phosphorylated tyrosine kinase fusion protein between Echinoderm microtubule-associated protein-like 1 (EML1) and Abelson 1 (ABL1) (51). When overexpressed in IL-3-dependent Ba/ F3 cells, EML1-ABL1 allows for growth factor-independent expansion of these cells through activation of survival and proliferation pathways, including Erk1/2, Lyn kinase and STAT5. These data further suggest the involvement of ABL1 fusions in the pathogenesis of some T-ALL cases, which may benefit from treatment with glivec/imatinib (51).

Activation of other STATs has been observed in human leukemias. In addition to STAT5, STAT1 and STAT3 appear to be constitutively activated in some ALL patient specimens (52). Moreover, methylation of SHP-1, a protein tyrosine phosphatase that acts as a negative regulator of the Jak/STAT pathway, was found in ALL primary cells and in the T-ALL cell line Jurkat, which may account for the constitutive activation of Jak and STAT3 (53). However, only STAT1 and STAT5 were reported to be constitutively activated in primary T-ALL (54). In addition to cell-autonomous lesions, activation of the Jak/STAT pathway may occur in response to cytokines and growth factors present in the leukemic microenvironment, thereby contributing to T-ALL disease progression. For instance, the 'pro-leukemic' cytokine IL-7 (22), was shown to induce Jak1, Jak3 and STAT5 activation in T-ALL cells (55). Altogether, and despite the evidence for Jak and STAT activation in some T-ALL patients and cell lines, Jak/STAT signaling has not been extensively demonstrated in patient specimens. Thus, the therapeutic potential of targeting this pathway remains largely speculative at this stage.

\section{TGF-ß}

Transforming growth factor-beta (TGF-ß) plays an important role in cellular homeostasis by regulating cell growth inhibition, cellular senescence, differentiation and apoptosis (56). Smad2 and Smad3, the principal cytoplasmic intermediates involved in the transduction of signals from TGF- $ß$ receptors, are activated when TGF- $ß$ binds to its cell-surface receptor and translocate from the cytoplasm to the nucleus, where they regulate gene transcription (57). While TGF- $ß$ is a potent negative regulator of hematopoiesis (58), the importance of down-regulated TGFB signaling in leukemogenesis has started to emerge.

Wolfraim and colleagues (59) described the loss of Smad3 in T-ALL pediatric patient cells, although no mutations in the Smad3 gene (MADH3) were found. Moreover, TALL cells display normal Smad3 mRNA levels, suggesting that Smad3 deletion occurs at the posttranslational level. Additionally, the authors showed that deletion of Smad3 can synergize with oncogenic events, such as loss of p27kip , in promoting T-cell leukemogenesis in mice. These data suggest that Smad3 may act as T-cell tumor suppressor and that defective TGF-ß signaling, either as a result of cell-intrinsic lesions or an altered microenvironmental context, may contribute to T-cell leukemogenesis. Strategies aimed at restoring Smad3 expression in T-ALL may prove beneficial, but are technically and clinically extremely challenging.

\section{Concluding remarks}

Alterations in signaling pathways play distinct roles in the etiology, maintenance and progression of T-ALL (Figure 1). Notch pathway is clearly deregulated in this type of leukemia. However, it is likely that Notch-signaling inhibitors (GSI compounds) will not be effective as single agents, and strategies involving their combination with drugs that inhibit other targets should be required. For example, chemoresistance to Notch inhibitors may be abolished by targeting the PI3K/Akt pathway (20). Additionally, our own data suggest that inclusion of inhibitors of PI3K pathway into current pediatric T-ALL therapeutic protocols may be of particular relevance. Despite the evidence for mis-regulation of MEK/ Erk, JAK/STAT and TGF-ß signaling pathways, involved in viability and proliferation, their exact relevance in primary $T$ ALL remains to be fully unraveled. Nonetheless, it is very plausible that the oncogenic signatures of some T-ALL cases embrace activation of these key pathways, and that those cases may benefit from tailor-made therapies involving the use of signaling-specific antagonists.

Overall, we suggest that the analysis of the intracellular signaling profile of T-ALL patients could not only serve to reveal novel molecular targets for treatment of this disease, but also to identify critical biomarkers for accurate and clinically relevant diagnosis and prognosis of T-ALL patient subsets. 


\section{Note added in proof}

JAK1 somatic activating mutations were very recently reported in ALL and particularly in adult T-ALL, where they account for $18 \%$ of the cases. JAK1 mutations appear to associate with poor response to therapy and reduced disease-free and overall survival (Flex E et al. Somatically acquired JAK1 mutations in adult acute lymphoblastic leukemia. J Exp Med 2008; 205: 751-758).

\section{References}

1. Pui $\mathrm{CH}$, Behm FG, Singh B, Schell MJ, Williams DL, Rivera $\mathrm{GK}$, et al. Heterogeneity of presenting features and their relation to treatment outcome in 120 children with T-cell acute lymphoblastic leukemia. Blood 1990; 75: 174-179.

2. Barata JT, Silva A, Brandão JG, Nadler LM, Cardoso AA, Boussiotis VA. Activation of PI3K is indispensable for interleukin 7-mediated viability, proliferation, glucose use, and growth of $\mathrm{T}$ cell acute lymphoblastic leukemia cells. $J$ Exp Med 2004; 200: 659-669.

3. Barata JT, Keenan TD, Silva A, Nadler LM, Boussiotis VA, Cardoso AA. Common gamma chain-signaling cytokines promote proliferation of T-cell acute lymphoblastic leukemia. Haematologica 2004; 89: 1459-1467.

4. Leong KG, Karsan A. Recent insights into the role of Notch signaling in tumorigenesis. Blood 2006; 107: 2223-2233.

5. Zweidler-McKay PA, Pear WS. Notch and T cell malignancy. Semin Cancer Biol 2004; 14: 329-340.

6. Ellisen LW, Bird J, West DC, Soreng AL, Reynolds TC, Smith SD, et al. TAN-1, the human homolog of the Drosophila notch gene, is broken by chromosomal translocations in T lymphoblastic neoplasms. Cell 1991; 66: 649-661.

7. Pear WS, Aster JC, Scott ML, Hasserjian RP, Soffer B, Sklar J, et al. Exclusive development of T cell neoplasms in mice transplanted with bone marrow expressing activated Notch alleles. J Exp Med 1996; 183: 2283-2291.

8. Feldman BJ, Hampton T, Cleary ML. A carboxy-terminal deletion mutant of Notch1 accelerates lymphoid oncogenesis in E2A-PBX1 transgenic mice. Blood 2000; 96: 19061913.

9. Hoemann CD, Beaulieu N, Girard L, Rebai N, Jolicoeur P. Two distinct Notch1 mutant alleles are involved in the induction of T-cell leukemia in c-myc transgenic mice. $\mathrm{Mol}$ Cell Biol 2000; 20: 3831-3842.

10. Weng AP, Ferrando AA, Lee W, Morris JP, Silverman LB, Sanchez-Irizarry C, et al. Activating mutations of NOTCH1 in human $T$ cell acute lymphoblastic leukemia. Science 2004; 306: 269-271.

11. Pear WS, Aster JC. T cell acute lymphoblastic leukemia/ lymphoma: a human cancer commonly associated with aberrant NOTCH1 signaling. Curr Opin Hematol 2004; 11: 426-433.

12. Eguchi-Ishimae $M$, Eguchi $M$, Kempski $H$, Greaves $M$. NOTCH1 mutation can be an early, prenatal genetic event in T-ALL. Blood 2008; 111: 376-378.

13. Gothert JR, Brake RL, Smeets M, Duhrsen U, Begley CG,

\section{Acknowledgments}

We thank Dr. Isabel Alcobia for a critical reading of the manuscript. We apologize to all the authors whose work, although of importance to the field, were not included in this review due to space limitations.
Izon DJ. NOTCH1 pathway activation is an early hallmark of SCL T leukemogenesis. Blood 2007; 110: 3753-3762.

14. Weng AP, Millholland JM, Yashiro-Ohtani Y, Arcangeli ML, Lau A, Wai C, et al. c-Myc is an important direct target of Notch1 in T-cell acute lymphoblastic leukemia/lymphoma. Genes Dev 2006; 20: 2096-2109.

15. Bellavia D, Campese AF, Alesse E, Vacca A, Felli MP, Balestri A, et al. Constitutive activation of NF-kappaB and Tcell leukemia/lymphoma in Notch3 transgenic mice. EMBO J 2000; 19: 3337-3348.

16. Talora C, Cialfi S, Oliviero C, Palermo R, Pascucci M, Frati $\mathrm{L}$, et al. Cross talk among Notch3, pre-TCR, and Tal1 in Tcell development and leukemogenesis. Blood 2006; 107: 3313-3320.

17. Yan $X Q$, Sarmiento $U$, Sun $Y$, Huang $G$, Guo J, Juan $T$, et al. A novel Notch ligand, DII4, induces T-cell leukemia/ lymphoma when overexpressed in mice by retroviral-mediated gene transfer. Blood 2001; 98: 3793-3799.

18. Lewis HD, Leveridge M, Strack PR, Haldon CD, O'neil J, $\mathrm{Kim} \mathrm{H}$, et al. Apoptosis in T cell acute lymphoblastic leukemia cells after cell cycle arrest induced by pharmacological inhibition of notch signaling. Chem Biol 2007; 14: 209-219.

19. Kogoshi H, Sato T, Koyama T, Nara N, Tohda S. Gammasecretase inhibitors suppress the growth of leukemia and lymphoma cells. Oncol Rep 2007; 18: 77-80.

20. Palomero T, Sulis ML, Cortina M, Real PJ, Barnes K, Ciofani $\mathrm{M}$, et al. Mutational loss of PTEN induces resistance to NOTCH1 inhibition in T-cell leukemia. Nat Med 2007; 13: 1203-1210.

21. Cantley LC. The phosphoinositide 3-kinase pathway. Science 2002; 296: 1655-1657.

22. Barata JT, Cardoso AA, Boussiotis VA. Interleukin-7 in T-cell acute lymphoblastic leukemia: an extrinsic factor supporting leukemogenesis? Leuk Lymphoma 2005; 46: 483-495.

23. Barata JT, Silva A, Abecasis M, Carlesso N, Cumano A, Cardoso AA. Molecular and functional evidence for activity of murine IL-7 on human lymphocytes. Exp Hematol 2006; 34: 1133-1142.

24. Kordes U, Krappmann D, Heissmeyer V, Ludwig WD, Scheidereit C. Transcription factor NF-kappaB is constitutively activated in acute lymphoblastic leukemia cells. Leukemia 2000; 14: 399-402.

25. Maser RS, Choudhury B, Campbell PJ, Feng B, Wong KK, Protopopov A, et al. Chromosomally unstable mouse tumours have genomic alterations similar to diverse human 
cancers. Nature 2007; 447: 966-971.

26. Luo JM, Liu ZL, Hao HL, Wang FX, Dong ZR, Ohno R. Mutation analysis of SHIP gene in acute leukemia. Zhong guo Shi Yan Xue Ye Xue Za Zhi 2004; 12: 420-426.

27. Yilmaz OH, Valdez R, Theisen BK, Guo W, Ferguson DO, $\mathrm{Wu} \mathrm{H}$, et al. Pten dependence distinguishes haematopoietic stem cells from leukaemia-initiating cells. Nature 2006; 441: 475-482.

28. Zhang J, Grindley JC, Yin T, Jayasinghe S, He XC, Ross JT, et al. PTEN maintains haematopoietic stem cells and acts in lineage choice and leukaemia prevention. Nature 2006; 441: 518-522.

29. Avellino R, Romano S, Parasole R, Bisogni R, Lamberti A, Poggi V, et al. Rapamycin stimulates apoptosis of childhood acute lymphoblastic leukemia cells. Blood 2005; 106: 14001406.

30. Barata JT, Cardoso AA, Nadler LM, Boussiotis VA. Interleukin-7 promotes survival and cell cycle progression of T-cell acute lymphoblastic leukemia cells by down-regulating the cyclin-dependent kinase inhibitor p27(kip1). Blood 2001; 98: 1524-1531.

31. Mungamuri SK, Yang X, Thor AD, Somasundaram K. Survival signaling by Notch1: mammalian target of rapamycin (mTOR)-dependent inhibition of p53. Cancer Res 2006; 66: 4715-4724.

32. Zunino SJ, Storms DH. Resveratrol-induced apoptosis is enhanced in acute lymphoblastic leukemia cells by modulation of the mitochondrial permeability transition pore. Cancer Lett 2006; 240: 123-134.

33. Uddin S, Hussain A, Al-Hussein K, Platanias LC, Bhatia KG. Inhibition of phosphatidylinositol 3'-kinase induces preferentially killing of PTEN-null T leukemias through AKT pathway. Biochem Biophys Res Commun 2004; 320: 932-938.

34. Lewis TS, Shapiro PS, Ahn NG. Signal transduction through MAP kinase cascades. Adv Cancer Res 1998; 74: 49-139.

35. Nishimoto $S$, Nishida E. MAPK signalling: ERK5 versus ERK1/2. EMBO Rep 2006; 7: 782-786.

36. Pearson G, Robinson F, Beers GT, Xu BE, Karandikar M, Berman $\mathrm{K}$, et al. Mitogen-activated protein (MAP) kinase pathways: regulation and physiological functions. Endocr Rev 2001; 22: 153-183.

37. Roux PP, Blenis J. ERK and p38 MAPK-activated protein kinases: a family of protein kinases with diverse biological functions. Microbiol Mol Biol Rev 2004; 68: 320-344.

38. Baer R, Hwang LY, Bash RO. Transcription factors of the bHLH and LIM families: synergistic mediators of T cell acute leukemia? Curr Top Microbiol Immunol 1997; 220: 55-65.

39. Lecuyer E, Hoang T. SCL: from the origin of hematopoiesis to stem cells and leukemia. Exp Hematol 2004; 32: 11-24.

40. Cheng JT, Cobb MH, Baer R. Phosphorylation of the TAL1 oncoprotein by the extracellular-signal-regulated protein kinase ERK1. Mol Cell Biol 1993; 13: 801-808.

41. Wadman IA, Hsu HL, Cobb MH, Baer R. The MAP kinase phosphorylation site of TAL1 occurs within a transcriptional activation domain. Oncogene 1994; 9: 3713-3716.

42. Garaude J, Cherni S, Kaminski S, Delepine E, ChableBessia C, Benkirane M, et al. ERK5 activates NF-kappaB in leukemic $T$ cells and is essential for their growth in vivo. $J$ Immunol 2006; 177: 7607-7617.

43. Kohno M, Pouyssegur J. Pharmacological inhibitors of the ERK signaling pathway: application as anticancer drugs.
Prog Cell Cycle Res 2003; 5: 219-224

44. Lu J, Quearry B, Harada H. p38-MAP kinase activation followed by BIM induction is essential for glucocorticoidinduced apoptosis in lymphoblastic leukemia cells. FEBS Lett 2006; 580: 3539-3544.

45. Rizzo MT, Pudlo N, Farrell L, Leaver A. Specificity of arachidonic acid-induced inhibition of growth and activation of cjun kinases and p38 mitogen-activated protein kinase in hematopoietic cells. Prostaglandins Leukot Essent Fatty Acids 2002; 66: 31-40.

46. Dreesen O, Brivanlou AH. Signaling pathways in cancer and embryonic stem cells. Stem Cell Rev 2007; 3: 7-17.

47. Valentino L, Pierre J. JAK/STAT signal transduction: regulators and implication in hematological malignancies. Biochem Pharmacol 2006; 71: 713-721.

48. Sinibaldi D, Wharton W, Turkson J, Bowman T, Pledger WJ, Jove R. Induction of p21WAF1/CIP1 and cyclin D1 expression by the Src oncoprotein in mouse fibroblasts: role of activated STAT3 signaling. Oncogene 2000; 19: 5419-5427.

49. Carron C, Cormier F, Janin A, Lacronique V, Giovannini M, Daniel MT, et al. TEL-JAK2 transgenic mice develop T-cell leukemia. Blood 2000; 95: 3891-3899.

50. Frantsve J, Schwaller J, Sternberg DW, Kutok J, Gilliland DG. Socs-1 inhibits TEL-JAK2-mediated transformation of hematopoietic cells through inhibition of JAK2 kinase activity and induction of proteasome-mediated degradation. $\mathrm{Mol}$ Cell Biol 2001; 21: 3547-3557.

51. De Keersmaecker K, Graux C, Odero MD, Mentens N, Somers R, Maertens J, et al. Fusion of EML1 to ABL1 in Tcell acute lymphoblastic leukemia with cryptic $t(9 ; 14)$ (q34;q32). Blood 2005; 105: 4849-4852.

52. Gouilleux-Gruart V, Gouilleux F, Desaint C, Claisse JF, Capiod JC, Delobel J, et al. STAT-related transcription factors are constitutively activated in peripheral blood cells from acute leukemia patients. Blood 1996; 87: 1692-1697.

53. Chim CS, Fung TK, Cheung WC, Liang R, Kwong YL. SOCS1 and SHP1 hypermethylation in multiple myeloma: implications for epigenetic activation of the Jak/STAT pathway. Blood 2004; 103: 4630-4635.

54. Weber-Nordt RM, Egen C, Wehinger J, Ludwig W, Gouilleux-Gruart V, Mertelsmann R, et al. Constitutive activation of STAT proteins in primary lymphoid and myeloid leukemia cells and in Epstein-Barr virus (EBV)-related lymphoma cell lines. Blood 1996; 88: 809-816.

55. Barata JT, Boussiotis VA, Yunes JA, Ferrando AA, Moreau LA, Veiga JP, et al. IL-7-dependent human leukemia T-cell line as a valuable tool for drug discovery in T-ALL. Blood 2004; 103: 1891-1900.

56. Shi Y, Massague J. Mechanisms of TGF-beta signaling from cell membrane to the nucleus. Cell 2003; 113: 685700.

57. Derynck R, Zhang YE. Smad-dependent and Smad-independent pathways in TGF-beta family signalling. Nature 2003; 425: 577-584.

58. Fortunel NO, Hatzfeld JA, Monier MN, Hatzfeld A. Control of hematopoietic stem/progenitor cell fate by transforming growth factor-beta. Oncol Res 2003; 13: 445-453.

59. Wolfraim LA, Fernandez TM, Mamura M, Fuller WL, Kumar $\mathrm{R}$, Cole DE, et al. Loss of Smad3 in acute T-cell lymphoblastic leukemia. N Engl J Med 2004; 351: 552-559. 